\title{
MODEL-BASED ADAPTIVE MONITORING: IMPROVING THE EFFECTIVENESS OF REEF MONITORING PROGRAMS
}

\author{
ABEYSIRI WICKRAMA LIYANAARACHCHIGE PUBUDU THILAN@
}

(Received 17 December 2021; first published online 9 February 2022)

2020 Mathematics subject classification: primary 62C12; secondary 62F35, 62M10, 62P12, 93C40.

Keywords and phrases: coral reefs, coral cover, environmental monitoring, adaptive monitoring design, time series models, semiparametric models.

Healthy ecosystems are at the heart of our survival on earth. They provide vital services and products such as maintaining the carbon dioxide level in the water, which would otherwise cause the ocean to become more acidic putting ocean life at risk. Coral reefs are an example of such ecosystems that support enormous biodiversity and are essential to the coastal populations surrounding them. In particular, they have a large economic impact through tourism, recreational activities and fishing. However, negative influences from human and environmental disturbances are seriously affecting the health of coral reefs globally. As a result, coral reef conservation has received considerable critical attention. In terms of conservation, reef monitoring programs play an important role in managing reef activities and providing information about the state of coral reefs, impacts of disturbances and vulnerabilities.

In the Great Barrier Reef (GBR), the largest coral reef system in the world, monitoring programs have been conducted for several decades. They are crucial tools used by policy makers and scientists to understand the condition and resilience of the reef. Although these monitoring programs have slightly changed over time, most of the survey sites have been fixed ensuring the capacity to study long-term trends that provide a clear picture about the changes in the reef. Despite this advantage, such monitoring programs are notoriously expensive to conduct, and there is interest in moving to more efficient methods to capture such information.

Adaptive monitoring approaches have been proposed recently for reef monitoring where survey locations can change over time depending on the reef health and disturbances while potentially preserving the capacity to study long-term trends. In this thesis, such adaptive design methods were further developed to improve the

Thesis submitted to Queensland University of Technology in April 2021; degree approved on 6 October 2021; supervisors James McGree, Helen Thompson, Patricia Menendez and Julian Caley.

(C) The Author(s), 2022. Published by Cambridge University Press on behalf of Australian Mathematical Publishing Association Inc. 
effectiveness of coral reef monitoring for collecting informative data while reducing the monitoring costs. For this purpose, the survey designs and the already collected data using such surveys at the GBR and Scott Reef, a coral reef system on the west coast of Australia, were considered and used as a baseline to assess new approaches to reef monitoring.

To that end, first, adaptive design methods were developed to improve long-term monitoring at the GBR. For this purpose, previously collected data using the Long-Term Monitoring Program (LTMP) of the GBR were used to develop a statistical model to predict coral cover, an indicator of coral health. This model was then used to guide adaptive surveying over time. Through such an approach, it was shown that capturing the spatial variability in coral cover and accounting for environmental disturbances, such as bleaching events, leads to more informative surveys. In doing so, it was also shown that the proposed approach to adaptive survey could yield twice the amount of information as recently proposed alternative approaches. Given such results, this work contributes to enhancing coral reef monitoring and more broadly ecosystem monitoring through reducing monitoring costs and collecting more informative data for achieving specified monitoring objectives [3].

Second, methods were proposed to estimate long-term trends in coral cover. Such work is of interest as, given adaptive designs may sample different sites/reefs/regions over time, it is likely that each location will not be sampled at fixed time points specified at the start of the survey. Given this, it is of interest to determine whether such survey approaches can be used to estimate long-term trends. For this purpose, coral cover data on the GBR were considered, and a coral growth model which can simultaneously capture coral growth and also decline due to environmental disturbances was developed. Based on this model, it was proposed that long-term trends can be assessed via the predictive distribution, and adaptive survey approaches were compared to surveying all locations at each time point. Here, it was shown that adaptive survey approaches can be used to maintain overall and reef level long-term trends in coral cover with little to no information loss when compared with surveying all locations in cases where no more than $50 \%$ and $20 \%$, respectively, of the survey effort was reduced. Accordingly, this research serves to further promote adaptive survey approaches in cases where long-term trends are of interest in ecological monitoring [2].

Third, an adaptive design framework was developed using semiparametric and interrupted time series models, and these proposed methods were used to guide future surveys at Scott Reef. This reef has been monitored for the last 27 years using the Scott Reef Research Program (SRRP). Through such monitoring, it has been observed that the reef experiences a variety of relatively frequent disturbances including cyclones and coral bleaching, and the interest was in determining whether survey practices should change (and adapt) into the future. To consider this, the historical data were used to develop a statistical model for the coral cover. Through this, it was found that the data were collected with unequal time gaps between observations, and also they exhibited nonlinear trends including sudden changes due to major disturbances such 
as mass bleaching events. Thus, flexible modelling approaches such as semiparametric models with interrupted time series approaches were considered to describe these data. Using these methods, on-going survey practices and recent changes to the survey practices at Scott Reef were assessed, and recommendations for future surveys at Scott Reef were proposed. Accordingly, it is hoped that the proposed designs and corresponding recommendations will have real benefit in monitoring the health of Scott Reef into the future [1].

In summary, this thesis contributes to enhancing coral reef monitoring using adaptive design methods in terms of reducing monitoring costs, improving knowledge capture and facilitating the evaluation of survey design practices.

\section{References}

[1] A. W. L. P. Thilan, R. Fisher, H. Thompson, P. Menendez, J. Gilmour and J. McGree, 'Adaptive monitoring of coral health on Scott Reef when data exhibit non-linear and disturbed trends over time', Preprint, 2021. https://www.authorea.com/users/429220/articles/532960adaptive-monitoring-of-coral-health-at-scott-reef-where-data-exhibit-nonlinear-and-disturbed-trends -over-time.

[2] A. W. L. P. Thilan, P. Menendez and J. McGree, 'Can adaptive design methods capture long-term trends in monitoring coral health?', Preprint, 2021. https://eprints.qut.edu.au/226955/.

[3] A. W. L. P. Thilan, E. E. Peterson, P. Menendez, J. Caley, C. Drovandi, C. Mellin and J. McGree, 'Bayesian design methods for improving the effectiveness of ecosystem monitoring', Preprint, 2021. https://eprints.qut.edu.au/131611/8/Thilan_et_al_2021_Bayesian_ design_method.pdf.

\footnotetext{
ABEYSIRI WICKRAMA LIYANAARACHCHIGE PUBUDU THILAN,

School of Mathematical Sciences, Faculty of Science,

Queensland University of Technology, Kelvin Grove,

Queensland 4059, Australia

e-mail: pubudu.liyanaarachchige@hdr.qut.edu.au
} 